\title{
FENÓMENO Y SUJETO: EL FUNDAMENTO DE LA PRESENCIA
}

\author{
Jacinto H. CALDERóN* \\ doi:10.11144/Javeriana.uph35-70.fsfp
}

\section{RESUMEN}

La filosofía, en su pretensión de ser un discurso ceñido a lo real, tiene que rendir cuentas a lo que en este artículo llamamos "presencia", es decir, a la condición fenoménica de todo ente que se presenta al psiquismo. En este sentido, encontramos que Kant, estableciendo su idealismo trascendental, se atiene a la presencia hasta que el sujeto aparece y lo presente parece cambiar de fundamento. En este momento aparece la doctrina de la ciencia de Fichte, planteando la presencia en otros términos que, de ser correctos, nos permiten acceder a una nueva dimensión de la filosofía.

Palabras clave: presencia; fenómeno; idealismo; sujeto; Fichte

Pontificia Universidad Javeriana, Bogotá, Colombia.

Correo electrónico: jacintocalderon@gmail.com

Para citar este artículo: CALderón, J. (2018). Fenómeno y sujeto: el fundamento de la presencia. Universitas Philosophica, 35(70), pp. 151-171. ISSN 0120-5323, ISSN en línea 2346-2426. doi:10.11144/Javeriana.uph35-70.dqpc 


\title{
PHENOMENON AND SUBJECT: THE FOUNDATION OF PRESENCE
}

\author{
Jacinto H. Calderón
}

\begin{abstract}
While philosophy pretends to be a discourse about reality as we perceive it, it has to pay tribute to "presence", as we call it in this article. "Presence" is every phenomenal condition of the beings which are presented to psychism. In this sense, we find that Kant, in his Transcendental Idealism, depends on "presence" until the real subject appears, and the "presented" seems to change its foundation. Fichte's Doctrine of Science, appeared at the time, defines "presence" in different terms; terms that, if correctly understood, grant us access to a new dimension of philosophy.
\end{abstract}

Keywords: presence; phenomenon; idealism; subject; Fichte 


\section{Introducción. La importancia del fenómeno}

LO QUE PRETENDEMOS ESTABLECER EN ESTE ARTíCULO tiene que ver con la idea general que encierra esta cita de Derrida (1989), que encontramos esencial para el comienzo de cualquier filosofar: "Nunca se puede exponer más de lo que en un momento determinado puede hacerse presente, manifiesto, lo que se puede mostrar, presentarse como algo presente, algo presente en su verdad, la verdad de un presente, o la presencia del presente" (p. 41).

La idea inmediata que encierra este corto pasaje es más que evidente: solo se puede hablar de lo que está presente; el fundamento de toda ciencia, en tanto que podemos hablar de ella, se encierra o debe encerrarse en un discurso sobre lo presente y manifiesto. En todo caso, es un estudio sobre el ente, dado que lo presente, como presente, es lo apareciente, es decir, el ente que se muestra. La ciencia, pues, debe tratar del ente. No debe separarse de él, pues en su compañía se desenvuelve como ciencia. Tal es la idea que se somete a juicio aquí.

Se trata, de un modo lejano, de seguir a Descartes (1995), en el sentido en que se refiere a lo claro y lo distinto:

Entiendo que es claro aquel conocimiento que es presente y manifiesto a un espíritu atento, tal y como decimos que vemos claramente los objetos cuando, estando ante nosotros, actúan con bastante fuerza y nuestros ojos están dispuestos a mirarlos. Es distinto aquel conocimiento que es en modo tal separado y distinto de todos los otros que solo comprende en sí lo que manifiestamente aparece a quien lo considera como es preciso (p. 48).

Para Descartes, la ciencia versa sobre lo claro y lo distinto, sobre lo que no podemos dudar, precisamente porque de lo que es claro y distinto no se puede dudar y, por ello, es digno de ser ciencia. Como tal, el argumento de lo que está presente es su propia presencia, tanto así como cuando Hegel (1993) nos recuerda que "comprender lo que es [Das was ist] constituye la tarea de la filosofía" (p. 59), es decir, bic Rhodus, hic saltus: lo claro, o sea, lo que es presente y manifiesto, ha de presentarse como el salto ante la muchedumbre: saltar frente a otro, manifestando allí tal capacidad -en el caso de la fábula de Esopo, el salto mismo-: 
Un atleta, que era muy conocido de sus conciudadanos por su debilidad, partió un día para tierras lejanas. Volvió después de algún tiempo, anunciando que había llevado a cabo grandes proezas en distintos países; contaba con especial esmero haber hecho en Rodas un salto que nunca antes ninguno de los atletas coronados en los juegos olímpicos había sido capaz de realizar, agregando además que presentaría los testigos de su hazaña si algunos de los que allí se hallaban presentes venían alguna vez a su tierra. Uno de los oyentes tomó la palabra y dijo:

-Oye, amigo: si eso es cierto, no necesitamos testigos; esto es Rodas, da el salto (Fábula 247, "El fanfarrón”).

No es lo mismo hablar del salto que presenciar el salto. Sostiene Marzoa (2003): “[...] obsérvese que el criterio de la claridad y distinción no quiere decir que lo que yo percibo clara y distintamente [...] 'exista' extramentalmente, en la 'realidad en sí' (aun suponiendo que esta noción tenga algún sentido)” (p. 41).

Asumiendo lo que hemos dicho en las líneas precedentes, no queda más remedio que definir lo que es científico a partir de lo presente manifiesto, aclarando, desde luego, que no es lo mismo "hablar del salto" que "dar el salto". Y no es lo mismo tampoco "oír hablar del salto" que "ver el salto".

El discurso, al menos de esta manera presentado, ¿ha de versar sobre lo que es presente y se muestra como presencia, o sea, como fenómeno (Erscheinung)? La respuesta ha de ser que sí, que efectivamente el discurso solo puede tratar lo que está ante mí; si de algo no puedo dudar es de la presencia en estado de presencia. Quizá los padres de la filosofía, Tales y Anaxímenes, tenían en mente algo similar, dado que, aplicando el criterio del salto, al intentar justificar el argumento del origen, respectivamente, por el agua o por el aire, en cierto sentido podían decir (simplemente): "mira”. Es decir, ponerlo ante la presencia. Son filósofos naturales, porque no se separan de la naturaleza, porque mantienen su argumento siempre al alcance de la mano.

El fenómeno como presencia es la presentación originaria de la filosofía, es decir, todo filósofo ha pretendido calibrar su discurso ante el otro haciéndolo pasar por presencia, por fenómeno, es decir, presentarlo con toda su claridad. De modo que no haya duda de que se puede "dar el salto" en cualquier momento. 
Pero hay un momento en el que se deja de mirar el salto para asegurar (sin problema) que lo presente es manifiesto sin necesidad de prueba. Las líneas que siguen son un intento de aclaración del motivo por el cual la filosofía del idealismo alemán se presenta como presencia, cuando en realidad tal cosa es manifiestamente imposible. A su vez, se pretende encauzar esta imposibilidad respecto del fenómeno a otro dominio en el que la filosofía extiende un ejercicio patente.

\section{El comienzo del filosofar: Kant}

A LO LARGO DEL SIGLO XVIII, ocurre una clara repulsa del pensamiento metafísico que se había originado con Descartes. Curiosamente, el filósofo reconocido por presentar lo claro y lo distinto como condición de la filosofía es tachado, hasta cierto punto, de no ser tan claro (amén de su continuador Spinoza), dado que, frente a la pura luz, tampoco se ve nada. La cuestión de la polémica reside, efectivamente, en que la claridad y la distinción se dirigen a instancias que no están a la vista, y por ello el concepto de realidad resulta ampliado.

Esta crítica, que se había originado con el primer empirista, Locke, encuentra su mayor exponente en Hume, que economiza la realidad a lo dado por los sentidos: "no hay más cera que la que arde".

Sin embargo, centremos la atención en Kant, en lo que refiere a nuestra búsqueda, en particular en la Crítica de la razón pura. Como seguidor, hasta cierto de punto, de Hume, le debe el haber despertado del "sueño dogmático de la razón”, es decir, de la conceptualización metafísica que no se atiene a la regla del fenómeno. Lo primero que nos indica Kant al comienzo de la Estética trascendental es lo siguiente: "[s]ean cuales sean el modo o los medios con que un conocimiento se refiera a los objetos, la intuición (Anschauung) es el modo por medio del cual el conocimiento se refiere inmediatamente a dichos objetos" (KRV A19, B33). De lo que se trata es, pues, de conocer y, por lo pronto, el conocimiento es lo que media entre el sujeto y el objeto, sin que, sin embargo, sea este la intuición; más bien, el conocimiento es la relación que mantiene el sujeto con el objeto a través de la intuición.

Notemos además la aclaración inmediata que realiza Kant a propósito del conocimiento y de la intuición: "tal intuición únicamente tiene lugar en la medida 
que el objeto (Gegenstand) nos es dado" (KRV A19, B33). Es decir que, acorde con la posición del texto de Derrida, la cuestión del filosofar inicia en la medida en que hay un objeto: lo que se hace presente, lo que lleva a la presencia es un objeto. Si no hay objeto, no hay conocimiento. Es cierto que para Descartes (1996) el uso de la intuición se puede traducir por ver, "la concepción de una mente pura y atenta” (p. 75), pero la intuición de la que habla aquí Kant, por de pronto, no es una indicación de la mente. Se trata de intuir lo que nos es dado, si no hay nada que se dé, no hay intuición. Sin embargo, la intuición no es posible sin un sujeto que intuya, e intuir ese sujeto es ya ir más allá de lo presente. La filosofía se escapa del "testimonio de los sentidos" en tanto que los utiliza, pero acorde al hilo kantiano, aquí no hay todavía intuición, excepto que el yo que intuye sea ese objeto. La filosofía, tal como la inicia Kant, trata del fenómeno, de lo que aparece, pero curiosamente, en el momento en que la posibilidad del aparecer aparece (amén de la redundancia) es también la posibilidad de que la filosofía marque la "regla del juego".

Por de pronto, Kant entiende que la forma de referirnos al objeto es precisamente la intuición. La intuición se refiere al "ente sensible" y relaciona el objeto con el entendimiento. De ahí que distinga inmediatamente dos facultades en el sujeto: sensibilidad y entendimiento, siendo en la primera, intuidos y, en la segunda, pensados. La referencia del entendimiento a la sensibilidad es constante, sin ella "ningún objeto se nos puede dar" (KRV A19, B33).

Dadas estas referencias, para Kant la forma de referirse al objeto (intuición) no trae de este el objeto como tal, sino el objeto indeterminado (unbestimmter Gegenstand $)^{1}$. La economización de la realidad nos aparece en la medida que no es el objeto lo que se ha intuido, sino algo indeterminado de él, el fenómeno, lo sensible o lo que aparece del mismo. Pareciera, desde ya, que todo conocimiento es una parte del conocimiento. No hay referencia a la totalidad del objeto sino únicamente a lo indeterminado de este. Es al lado determinado del objeto, parece, a lo que no podemos llegar. De ahí la distinción, inmediata, entre fenómeno y cosa en sí.

La cosa en sí, de todas formas, es lo que se aspira conocer. El comienzo del filosofar quiere ajustarse a lo presente, pero de ser así, y siendo kantianos, cabe 
decir que lo que logramos no es más que el fenómeno y el anhelo de la cosa en sí. Sin embargo, Kant es más concesivo de lo que parece en lo que respecta a otorgar realidad al conocimiento. Por de pronto, en el paso de la sensibilidad al entendimiento nos aparecen dos elementos que nos abren las puertas hacia el concepto: la representación y la intuición pura.

La representación ha de entenderse (a falta de definición explícita) como la forma del fenómeno, en la medida que éste se ha despojado de su materialidad y, en la medida que la representación se hace presente, es la intuición pura la que se hace cargo de operar con ella. El tránsito es el siguiente, como vemos: el objeto, lo indeterminado del objeto, la forma de lo indeterminado del objeto. En este sentido, Schopenhauer no tuvo que preocuparse mucho de la reacción de la crítica cuando exclamó aquello de que "el mundo es mi representación". Lo que sea el mundo es lo que cae bajo mi representación, que es lo interesante del caso.

A su vez, lo que decimos que es el mundo (el "mi" de la representación schopenhaueriana) es lo que se quiere expresar aquí, a saber, que es el sujeto quien sitúa lo presente (la verdad de un presente) bajo sus propias condiciones. La intuición es dada a priori en el psiquismo y toma del objeto la representación. La representación es por ello, un ser-para-otro, no es algo por sí misma sino que es algo para el entendimiento, facultad que, situada más allá de la sensibilidad, otorga las condiciones de toda experiencia posible.

Por de pronto, entonces, tenemos que para Kant la epistemología no es hacerse cargo de lo presente en cuanto presente sino de demostrar cómo es posible el conocimiento y la experiencia del mismo, es decir, cómo los conceptos conectan con las representaciones y, a su vez, cómo estas conectan con los fenómenos.

Sin pretender realizar aquí un estudio detallado de la Estética trascendental y de la Lógica trascendental, lo cierto es que hemos de aproximarnos a lo que en verdad ha de ser presente para el filósofo, habida cuenta de que estamos alejándonos de lo presente tal como se pedía al principio. ¿O es posible que lo que intentemos hacer aquí sea precisamente extender lo presente (la verdad del presente)?

3. La tarea del entendimiento y la aparición del sujeto

LO QUE SEA LA COSA EN SÍ ES LO QUE BUSCA TODO FILOSOFAR, ahora bien, "entre la cosa en sí y su fenómeno no se da continuidad sino un salto" (Rivera de 
Rosales, 2011, p. 30). Salto que, por cierto, no se puede ver ni dar, solo (pareciera) perderse de vista. La filosofía, por ello, parece renunciar al juego, es decir, renunciar a lo que precisamente es. En términos kantianos, además, hemos de resignarnos a no poder conocer la cosa en sí: solo conocemos fenómenos. Pero siendo la exposición kantiana insuperable en muchos sentidos, no podemos sino mantenernos en el juego del entendimiento sabiendo que nuestro saber no alcanza a la cosa en sí, con una inevitable renuncia a la metafísica, porque de esta no podemos hacer ciencia: "las cosas en sí mismas, fuera de toda experiencia, no serían nada para mí, no podrían ser objetos míos” (Rivera de Rosales, 2011, p. 32).

El entendimiento (más allá de la sensibilidad) es el encargado de dirimir lo que se puede obtener de lo presente y lo que no. La labor del entendimiento es necesariamente la labor de la distinción entre lo posible y lo no posible²: "Hay que dejar en todo rigor aparecer/desaparecer la huella de lo que excede la verdad del ser. Huella (de lo) que no puede nunca presentarse, huella que en sí misma no puede nunca presentarse: aparecer y manifestarse como tal en su fenómeno" (Derrida, 1989, p. 57).

Lo cierto es que este pasaje de Derrida va dirigido más bien a un texto; no obstante, la dificultad en relación con el texto es similar a la dificultad del conocer que deja, a su vez, huellas del noúmeno, del que podemos decir que es lo que se presenta sin presentarse, "la presencia de una ausencia".

Pero hemos avanzado lo suficiente para poder dar un salto que nos ha de situar más allá del fenómeno. En efecto, la sensibilidad ha consistido en el paso de la presencialidad del objeto a su fenómeno y, este, merced a las formas a priori de la sensibilidad, espacio y tiempo, ha sido ordenado según un criterio. Como podemos ver, lo que estamos haciendo aquí no es otra cosa que una teoría del conocimiento. Pero esta teoría tiene sentido únicamente para un sujeto que, por un lado, tenga la necesidad de conocer y, por otro, pueda validar el conocimiento que le llega.

Es necesario, por eso mismo, que aparezca un sujeto que pueda dar sentido al conocimiento. El sentido que el sujeto da al conocimiento puede ser subjetivo u objetivo. Subjetivo, porque el sujeto es quien da las condiciones al objeto.

2 Pero no imposible, porque no es lo mismo no llegar a conocer que negar que lo que no podemos conocer no exista. 
Objetivo, porque la experiencia del conocimiento ha de validarse ante el objeto. El sujeto, al menos en este primer momento, es la significación real del conocimiento. ¿Qué significa un objeto para un sujeto ?3 Lo que se busca es el conocimiento del objeto, pero ahora hemos de atender más a la búsqueda del sujeto, que es lo que nos va a permitir entrever, finalmente, una eminente cuestión filosófica:

Nuestro conocimiento surge básicamente de dos fuentes del psiquismo: la primera es la facultad de recibir representaciones (receptividad de las impresiones); la segunda es la facultad de conocer un objeto a través de tales representaciones (espontaneidad de los conceptos). A través de la primera se nos da un objeto; a través de la segunda, lo pensamos en relación con la representación (KRV A50, B74).

Kant, en esta cita, ha mostrado su juego y ha puesto las cartas encima de la mesa: el conocimiento es un juego entre conceptos e intuiciones, de tal suerte que estas últimas "sin conceptos, son ciegas" (KRV A51, B74). Pero el concepto, ¿qué es? Kant deja apoyado el conocimiento en la sensibilidad, en cuyo caso ha dejada intacta la cita de Derrida, con la que comenzaba este escrito. Pero si quiere seguir aportando conocimiento tiene que hacer un giro, o, si se prefiere, cambiar el juego para mostrar lo siguiente: "El yo pienso tiene que poder acompañar a todas mis representaciones" (KRV B132).

Para Kant, una analítica de los conceptos consiste en "investigar la posibilidad de los conceptos a priori a base de buscarlos solo en el entendimiento como su lugar de procedencia", que supone para Kant "la tarea propia de una filosofía trascendental" (KRV A66, B90).

Por tanto, la filosofía es precisamente demostrar que cuando hablamos de algo (algo presente) ese algo tiene una justificación. El entendimiento kantiano es la fuente de toda filosofía, dado que aquí no solo se piensa el objeto, sino que se juzga sobre su condición de validez:

[...] pero la facultad de juzgar (sin la cual no habría, propiamente hablando, conocimiento) es la facultad de aplicar esas reglas o [...] la capacidad de ligar un sujeto y un predicado, de vincular un antes con un después, que es sin más

3 ¿Qué significa un sujeto para un objeto? La pregunta, en este orden, no sería más que una impertinencia, pues en ese terreno no podemos meternos, por mucho que lo intentemos. 
lo que vulgarmente denominamos "sano entendimiento" o "sentido común" (Pardo, 2004, p. 104).

Aplicar las reglas es la tarea del entendimiento y la regla que generalmente se aplica es la que relaciona al sujeto con el objeto, la regla que supone que lo que decimos del objeto es su presencia. La regla del entendimiento es relacionar conceptos con representaciones y estas con objetos. Pero solo se puede hacer filosofía si los objetos que buscamos relacionar tienen presencialidad, es decir, si se han mostrado como fenómenos.

Los límites de lo que es posible conocer necesitan de una condición de posibilidad para que puedan ser puestos. Pues, en efecto, aquello de lo que tenemos experiencia es la posibilidad del conocimiento, pero aquí la principal cuestión que nos ocupa es entender que el conocimiento es para alguien y no para algo; ese "para alguien" que conoce y da la regla es el sujeto.

\section{Sujeto trascendental e idealismo}

Para Kant hay una CUestión ineludible en el conocimiento, a saber, "cómo pueden tener validez objetiva las condiciones subjetivas del pensar" (KRV A89, B122). Sin embargo, no se ha hablado de subjetividad hasta que hemos ido más allá del fenómeno, en cuyo caso tenemos que la esencia del giro copernicano es que es el sujeto quien pone las reglas al objeto. Lo subjetivo es lo necesario en el conocimiento, pero a su vez lo subjetivo es ahora el escándalo, pues o bien la presencia cambia para devenir algo distinto (y más rico) o bien el discurso filosófico no puede ir más allá de la representación.

La clave la encontramos en el concepto de experiencia (Erfahrung), concepto clave para la filosofía kantiana, pero también para la filosofía de Hegel: ees posible tener una relación objetiva del sujeto al objeto? De ser posible, tal cosa es la experiencia. Ahora bien, el fundamento de la experiencia no lo encontramos en la suposición de la realidad del mundo, en su presencia, sino en la presencia del sujeto que posibilita la experiencia misma, merced a sus facultadas a priori, así como a la labor del entendimiento. La deducción trascendental de las categorías revela la conexión del entendimiento con el fenómeno, la posibilidad de la experiencia como tal. 
Pero Kant daba la impresión de haberse olvidado algo en la primera edición de la Crítica de la razón pura, a saber, explicitar al sujeto del conocimiento, o sea, al yo. Por ese motivo, al empezar la deducción de las categorías según la segunda edición encontramos al sujeto, más o menos explícitamente:

El yo pienso tiene que poder acompañar todas mis representaciones. De lo contrario, sería representado en mí algo que no podría ser pensado, lo que equivale a decir que la representación, o bien sería imposible o, al menos, no sería nada para mí (KRV B132).

Kant había definido la síntesis - la capacidad de unificar las representaciones en un concepto- como un "acto intelectual" (Verstandeshandlung). Este acto solo cobra sentido si, efectivamente, un sujeto lo realiza. De no ser así, o bien no pensaría el sujeto en cuestión (sería como una maquina) o alguien estaría pensando por él. Si no hay representación del sujeto no hay representación alguna. El sujeto es la posibilidad de cualquier conocimiento, aquello que de no estar presente negaría cualquier presencialidad. Pero el yo pienso es la representación que acompaña a toda representación; por eso, Kant define la apercepción pura $\mathrm{u}$ originaria como una autoconciencia que da lugar a la representación yo pienso. Pensar significa conciencia de estar pensando: "la conciencia empírica que acompaña representaciones diversas es, en sí misma, dispersa y carece de relación con la identidad del sujeto" (KRV B133).

Lo que entra en juego ahora es determinante para la consideración de toda presencialidad, es decir, de todo conocimiento: el pensar y la representación del pensar son dos cosas distintas, así que, por un lado, tenemos lo que representa (el yo pienso) y, por otro, lo que es representado. Para que haya autoconciencia ha de haber identidad de la misma. A su vez, para que haya conocimiento ha de hacerse presente la condición del conocer. Fichte (1997), haciendo acopio de la tarea que empieza Kant al demostrar el conocimiento, entiende que la filosofía no es otra cosa que responder a esta cuestión: “ ¿ $[$ c] uál es la razón de ser del sistema de representaciones acompañadas de un sentimiento de necesidad, y de este sentimiento mismo?” (p. 8). La filosofía es, pues, hacer deducción trascendental, de donde se extrae la idea, nada nueva, de que el idealismo alemán surge como un comentario a la deducción trascendental kantiana, comentario que quiere poner de relieve la necesidad interna de toda filosofía de hacer presente al que posibilita 
lo presente, sea este el sujeto trascendental kantiano, el yo de Fichte o el espíritu absoluto hegeliano.

Para Fichte la cuestión no entraña, de todas formas, demasiado misterio, puesto que lo que ha aparecido en estas líneas no es más que el intento de comprensión del conocimiento sin sujeto, hasta que el entendimiento deja claro que el conocimiento es conocimiento para un sujeto. Dicho de otra manera, para Kant la introducción del sujeto significaba dejar de lado el fenómeno extraído de la sensibilidad para ocuparse del fenómeno que proviene de la intuición interna. Por eso, Fichte (1975), que no consideró necesario hacer una novela de intriga para hacer filosofía, nos dice al comenzar su Fundamento de toda doctrina de la ciencia de 1794: "[a]sí, pues, el fundamento que explica todos los hechos de la conciencia empírica [es decir, lo que hace posible un conocimiento determinado] es el siguiente: que antes de poner algo en el Yo, el Yo mismo sea puesto" (p. 16).

Para Fichte (y también para Kant) la verdad del yo pienso no se presenta como lo claro y distinto cartesiano (que también lo es), sino como el fundamento del psiquismo. Para Fichte, el yo es la piedra angular de la filosofía y este se revela como el fundamento de la presencia, aquello sin lo cual no hay conocimiento, ni nada en absoluto.

Kant, una vez establecido el yo pienso y la identidad de la conciencia, prosigue su filosofía teórica observando todos los detalles en los que el yo pienso puede acompañar a sus representaciones. La filosofía se hace tal en la medida que se puede demostrar la experiencia, es decir, cuando los objetos concuerdan con las representaciones, o bien, en los términos de Fichte, cuando las representaciones que tienen un sentimiento de necesidad concuerdan con sus objetos.

La filosofía tiene ahora que demostrar la experiencia posible, lo que equivale a determinar lo que es real (sujeto a la experiencia) de lo que no es (lo que no está sujeto a una experiencia posible). Para Fichte (1997), además, "si los resultados de una filosofía no coinciden con la experiencia, seguro que esa filosofía es falsa" (p. 33).

Es fácil suponer que Kant estaría perfectamente de acuerdo con esta idea, puesto que su filosofía, en tanto que crítica de la razón, pretende sentar precisamente el criterio de la experiencia, para que el pensamiento humano no se desboque. Y por este motivo, Fichte asume que la filosofía no es otra cosa que 
demostrar cuál es la razón de ser de las representaciones que coinciden con sus objetos, o bien las representaciones que solo pueden ser de una determinada manera. En suma, filosofía es deducir las categorías, demostrar la experiencia posible, atender y comprender la realidad misma.

El caso es que, si bien Kant y Fichte coinciden en la razón del quehacer filosófico, es necesario examinar cuál es, para ellos, el criterio de la experiencia posible. Es decir, la filosofía consiste en demostrar la experiencia; ahora bien, el fundamento por el cual esa experiencia puede ser algo para el sujeto es ahora la clave: ¿cuál es el fundamento sobre el que apoyar toda experiencia posible?

Se trata del sujeto trascendental. Y la mejor forma de verlo no es más que atender a la idea kantiana de que "los pensamientos sin contenido son vacíos; las intuiciones sin conceptos son ciegas" (KRV A51, B75). Si no hay entendimiento, no hay presencialidad alguna, pues el criterio de lo presente, aunque reside en lo presente mismo, necesita ser entendido. No tiene sentido alguno hablar de conocimiento si no hay sujeto que tenga el cometido de conocer. La distinción entre representaciones y conceptos es la distinción, a su vez, entre sensibilidad y entendimiento. Claro, las intuiciones sin conceptos no tienen adónde ir. En otros términos, el nivel de la percepción (o sea, donde el mundo queda representado) se distingue del nivel de la apercepción (la autoconciencia) (Rivera de Rosales, 2011, p. 128).

Así pues, el saber que vuelve sobre sí tiene que ser la clave del conocimiento mismo; sin embargo, aquí hay encerrado un problema que es idéntico al del conocimiento sensible, pero en el nivel del entendimiento: "ese saber de sí comporta, como toda conciencia real, una dualidad objeto-sujeto, un yo en cuanto objeto conocido y un yo en cuanto sujeto cognoscente" (Rivera de Rosales, 2011, p. 129). El criterio del conocer es la presencia y por ello el discurso kantiano tiene el deber de traer a la presencia al sujeto trascendental; también, en este sentido, Fichte tiene todo el derecho de hacer continuar la filosofía por el Yo puro, como fundamento de todo lo real.

El problema aquí reside en que tomar como presencia al sujeto implica objetivarlo, es decir, representarlo y, a su vez, verificar que lo representado es lo representante. Este juego filosófico va a ser la puerta de entrada al idealismo. Pero claramente vemos que el yo representante o cognoscente no es tan fácil de asir como parece, de ahí que Schopenhauer (2010) dijera que "aquello que lo conoce 
todo y no es conocido por nadie es el sujeto" (p. 116). La apercepción trascendental es el fundamento de todo conocimiento, pero una comprensión de esta no es tan sencilla. El propio Kant, cuando emprende el estudio de la Deducción trascendental de las categorías según la segunda edición, pretende ser claro (más que en la primera edición, desde luego, donde el sujeto quedaba implícito), pero no se puede decir que lo consiga del todo.

En realidad, distinguir entre el sujeto representado y el sujeto trascendental es necesariamente confuso, porque estar en la completa oscuridad es lo mismo que estar en la pura luz, y sujeto trascendental es, sencillamente, la luz del entendimiento, y en cuanto luz no puede ser mirado directamente.

Que haya conocimiento supone la unidad de la conciencia, pero ser conscientes de la unidad de la conciencia es suponer a esta de un lado distinto, del lado de lo representado. La autoconciencia requiere de su unidad y esta es lograda para Kant mediante una síntesis a priori, que le revela a la conciencia su ser-para-sí.

\section{Idealismo}

Fichte establece como motor de su sistema al Yo, que en el momento que nos ocupa es la unidad de la conciencia, o el que conoce todo, pero no se deja conocer. El Yo de Fichte no queda anclado en el plano del conocimiento, necesita de la praxis para ser descubierto. En cambio, para Kant, la unidad de la conciencia queda situada en el ámbito de la síntesis del conocimiento. El sujeto trascendental kantiano es un yo que piensa. El Yo de Fichte es un yo que actúa.

Lo que inicia el idealismo es, precisamente, entender que la filosofía encuentra un nuevo objeto (aunque huelga decir que el objeto aquí es el sujeto) y este objeto abre la realidad más allá de la economía de la verdad. El hallazgo del sujeto trascendental es situar la filosofía en el elemento de lo que fundamenta todo presente, más allá del ente, en el territorio del ser.

No es objeto de este artículo explicar el idealismo de Fichte, sino únicamente limitarse a la comprobación de si lo que es su puerta de entrada es una puerta válida. Retomando la frase de Derrida que citamos al inicio de este artículo, cuando refiere que lo que busca la filosofía es poder develar el sentido de esta idea: "la verdad de un presente, la presencia de un presente", encontramos que 
Fichte tiene como tarea inmediata comprobar si el Yo al que hacemos referencia responde a la exigencia de la presencialidad.

Para tal evento Fichte ha de observar cuidadosamente el fenómeno para verificar su relación con el conocimiento. Por lo pronto, ya nos hemos referido a las representaciones que se acompañan con un sentimiento de necesidad, esto es, aquellas que el intelecto no puede modificar sin hacer una afrenta al conocimiento.

Ahora bien, Fichte distingue en la representación una parte que corresponde al sujeto y otra al objeto. La pregunta es evidente: ¿cuál de los dos elementos ofrece más claridad a la hora de fundamentar el conocimiento? ¿El sujeto o el objeto? El criterio de la presencia necesita de algo que esté presente y eso es a lo que llamamos el ente. Sin embargo, el fundamento del ente ¿̇dónde ha de ubicarse?

A su vez, Fichte en cierto sentido asimila la pregunta formulada por Leibniz (1940): “¿por qué existe algo más bien que nada?” (p. 64), y formulada más adelante por Heidegger (2001): “¿por qué es ente y no más bien la nada?” (p. 11). Se pregunta Fichte (1997): “¿cómo llegamos a admitir un ser?” (p. 42).

La representación y el fenómeno necesitan del criterio de la presencia, pues el fenómeno es fenómeno bajo la consideración del sujeto, y la representación es la forma de este. Pero aun así hay una cuestión evidente: ¿cuál es el fundamento del fenómeno? Necesariamente hemos de admitir un ser no presente para nosotros. La pregunta filosófica “ipor qué algo es algo?” pretende hallar este ser.

Es aquí donde Fichte puede plantear ya su idealismo en los términos de la pura presencia, en el sentido de que o bien nos quedamos a ciegas, o bien podemos aclarar algo, de la cuestión de la presencia en el fenómeno. En efecto, hecha la abstracción ${ }^{4}$ entre el yo y la cosa (el sujeto y el objeto), sitúo como fundamento del conocimiento al objeto, cayendo en lo que Fichte desdeñosamente llama dogmatismo, o, por el contrario, sitúo como fundamento al sujeto, a lo que denomina idealismo. En otros términos, se ha de elegir (y Fichte habla aquí más bien de elección) entre un yo en sí o una cosa en sí.

Por de pronto, queda claro que, para Fichte, el fundamento de la presencia no está en la presencia misma, sino en lo que más allá de ella la hace posible. La

4 Fichte (1997) entiende por abstracción lo siguiente: "separar, mediante la libertad de pensamiento, lo que en la experiencia se da unido" (p. 10). 
presencia, según esto, no es más que apariencia (el phántasma). Pero si la filosofía ha de verificar lo presente como presente, ¿no estamos defraudando a nuestro propio interés, es decir, no se está cometiendo un error?

El idealismo que inaugura Fichte debe justificarse ante este error. Sin embargo, el error no es para Fichte un problema nuevo; de hecho, su filosofía pretende salvar a la filosofía de un error ya viejo, el error de la cosa en sí. Como tal, el presumible error del conocimiento era ir más allá de la presencia. Sin embargo, o la presencia era algo por sí (el fenómeno como la realidad aprehensible, pero a su vez la única realidad) o la presencia se derivaba de otra cosa (la cosa en sí). Pues bien, de la cosa en sí no podemos decir nada; si algo dejó claro Kant en su primera crítica fue precisamente esto. Pero, entre los que desde temprano empezaron a criticar a Kant (Obereit, Jacobi, etc.) le acusaban continuamente de ignorar la cosa en sí, cuando en realidad era esta la que posibilitaba toda la filosofía. A su vez, lo que se busca es un fundamento real (a su vez, presente, manifiesto) del conocimiento. Para Fichte (1997) la cuestión es evidente: la cosa en sí no puede ser el fundamento del conocimiento sencillamente porque la cosa en sí no es nada, "es una pura invención y no tiene realidad alguna" (p. 13). ¿Por qué motivo, entonces, se pretende situar el fundamento en algo que no tiene realidad? El problema, ciertamente de visión, es la esencia del dogmático que, ajeno a un pensar frío (la seriedad del concepto que pedía Hegel) se deja llevar por el entusiasmo. Entusiasmo que para Fichte no conduce a poder vislumbrar el fundamento del conocimiento, antes bien, lo oscurece.

De la cosa en sí nada se sabe, en cambio del yo en sí podemos expresar algo. Para Fichte, la cuestión radica en preguntarse sobre la presencia de los dos fundamentos: ¿qué ha de aparecer como más real? Hegel dirá más adelante que la cosa en sí es determinada como el "más allá de la conciencia". Y estando más allá de la conciencia, está fuera de toda determinación: entre no saber nada y no poder decir nada, permanece la cosa en sí.

Fichte (1997), desde luego, se gana la acusación de nihilismo. La cosa en sí no es nada, "no se da precisamente en la experiencia, pues el sistema de la experiencia no es otra cosa que el pensamiento acompañado del sentimiento de necesidad" (p. 13). Pero el caso es que, si bien se pone sobre la mesa la acusación, la opción de Fichte no es tampoco gratuita: no se puede poner como fundamento del conocimiento a lo que no se puede conocer, a la ausencia de 
la presencia. Por consiguiente, o es el yo quien fundamenta el conocimiento, o lo es la nada.

\section{El yo y la intuición intelectual: el problema con Kant}

Para Fichte, la única filosofía posible es el idealismo, es decir, la filosofía que sitúa al sujeto como fundamento de la presencia. ¿Qué quiere decir esto? Sencillamente que el objeto de la filosofía se hace presente por primera vez. $\mathrm{Al}$ igual que pasaba con Descartes, que llega al sujeto por una deducción, y con Kant, que ponía al yo pienso como el acompañante de la representación, Fichte sitúa al Yo en sí, pero ya no como el resultado de una deducción ni de un acompañamiento, sino como fruto de una intuición, o sea, de un ver.

Descartes estaría perfectamente de acuerdo con Fichte. Llegan a la misma conclusión: el sujeto es el fundamento. Pero con Kant la situación no permanece tan clara. El problema aparece en la intuición. Kant había admitido la posibilidad de tres formas de intuición y la realidad de dos. Intuición empírica e intuición pura, que pertenecen al terreno del fenómeno y de la representación. Y la intuición intelectual, que crea forma y género y es la propia de Dios, en ningún caso la del hombre.

Sea como fuere, la intuición es "el modo de referirse a los objetos", por lo cual hay intuición si hay objeto, o sea presencia. No podemos intuir la cosa en sí, desde el punto de vista kantiano, porque nuestro intuir se queda corto, pero tampoco desde el punto de vista de Fichte, dado que la cosa en sí no es nada (y no se puede intuir la nada).

Fichte (1982) necesita que una intuición le muestre algo, pero, a fuerza de ser kantiano, acepta que la intuición no puede mostrar al yo como fenómeno, es decir, que le podríamos conocer por sus efectos, pero no se podría decir nada de él a priori. Su solución estriba, entonces, en que la intuición no se dirija a un fenómeno, sino que se dirija a un actuar: "evidentemente hemos de tener un principio fundamental real y no meramente formal, pero tal principio no tiene por qué expresar precisamente un hecho [Tatsache], puede expresar también una acción [Tathandlung]" (p. 66). En todo caso, la pregunta aquí sería: ¿podemos intuirnos a nosotros mismos? De ser así, esta intuición no podría ser solo fenoménica. Pero claro, de intuirnos a nosotros mismos, ¿nos intuiríamos como el ser de Parménides, inmóviles y redondos, o nos intuiríamos en nuestro propio 
devenir, o sea, en el pleno movimiento? Para Fichte el yo es concebido en su actividad; tanto es así, que el simple hecho de representarlo incluye su propia libertad. Se le intuye produciéndose a sí mismo.

Pero es una intuición intelectual la que pone al Yo en el juego. Ahora bien, no es una intuición intelectual entendida en sentido kantiano, sino que es una intuición dirigida a acciones; por ello, "este actuar es precisamente el concepto del yo, y el concepto del yo es el concepto de este actuar" (Fichte, 1997, p. 46).

No hay filosofía sin sujeto, y la experiencia muestra que si algo hay que sea presente en el conocer es el yo que se configura a sí mismo. No hay filosofía sin sujeto. El sujeto es la condición de todo presente y lo presente mismo. Si no está presente, no hay presencia. La intuición intelectual es la condición real del fundamento del presente:

No puedo dar un paso, ni mover la mano o el pie, sin la intuición intelectual de mi autoconciencia en estas acciones; sólo mediante esta intuición sé que yo lo hago, sólo por ella distingo mi actuar, y en éste me distingo a mí mismo del objeto del actuar que se me ha hecho presente. Todo aquel que cree tener una actividad alega esta intuición. En ella está la fuente de la vida, y sin ella lo que hay es la muerte (Fichte, 1997, p. 50).

Es claro que, para Fichte, es imposible suponer cualquier presencia sin que su fundamento esté presente. No obstante, ¿no tendría también un problema de economización de la realidad? La reducción es la pérdida de la cosa en sí, pero el fenómeno se mantiene, sigue siendo lo que es: algo para un sujeto. No hay pérdida de realidad si tenemos en cuenta que la posibilidad de la cosa en sí era irreal: esta es una invención, una pura nada. El sujeto, como fundamento de lo presente, y lo presente mismo, son el fundamento de lo real. Pero para que Fichte pueda desarrollar su filosofía tiene que demostrar que una intuición dirigida a un actuar tiene validez por sí misma. Para Fichte, como ya hemos visto, la intuición no necesita de mucha explicación, dado que sin ella no hay sujeto, y sin sujeto no hay dónde hacerse presente.

La filosofía de Fichte puede establecerse como tal, o sea, como filosofía verdadera, en la medida que haga conectar la Doctrina de la ciencia con el idealismo trascendental. De hecho, la demostración de la intuición intelectual no parece tan importante como el hecho de que Kant pueda estar de acuerdo con él. No 
obstante, es clara la insistencia de Fichte en que sin tal intuición no hay sujeto y, sin él, no hay filosofía alguna.

Pues bien, la primera consecuencia de cara al fundamento de toda presencia es que tal fundamento ha de mantener un diálogo con Kant y, sea como fuere, no oponerse a algunas de sus conclusiones. Convertir a Kant, entonces, en el modelo del arbitraje despeja toda duda de por dónde debe ir la filosofía.

En segundo lugar, equé tan de acuerdo estaría Kant con la doctrina de la ciencia? Fichte, desde luego, tiene claro que su doctrina es coherente con el idealismo trascendental, e, incluso, considera que la filosofía debe seguir por su camino. Para ello, en la Segunda introducción a la doctrina de la ciencia, dedica bastantes páginas a explicar por qué esta es consistente con el idealismo kantiano, no lo contradice e, incluso, amplía sus resultados, es decir, permite un aumento del logos y permite presenciar el absoluto.

Kant parece dar en un primer momento una aprobación crítica, basado en las noticias que tuvo de Fichte e incluso en la correspondencia que mantuvieron ${ }^{5}$. En la carta dirigida a Fichte, escrita en Jena en el otoño de 1797, dice Kant (2011):

Vuestros trabajos, [...] me fueron enviados en 1795 y 1796 [...] veo que en vuestras nuevas piezas se desarrolla vuestro excelente talento para una exposición vivaz unida la popularidad, con las cuales habéis recorrido la espinosa senda de la escolástica, y no encontraréis necesario volver a verla de nuevo (p. 85).

Lo cierto es que le da ánimos, pero, a su vez, ¿qué tanto habría leído Kant a Fichte? Por eso, según el testimonio de Johann Friedrich Abbeg en junio de 1798, quien lleva a Kant una misiva de Fichte, relata que el ya anciano Kant (2011) le contesta, después de haberla leído:

Esto es un gran cumplido, también me escribe obsequiosamente, pero se me cuela un amargor, y es que yo no logro aclararme sobre él o declararme por él: y nada se arreglará porque él imagine todo tan bonito. No leo del todo sus escritos, pero recientemente leí la recensión de estos en la Jenaische Literaturzeitung, la primera vez no supe muy bien qué es lo que él quería; lo leí por segunda vez y creí poder entender algo, pero no. Él sostiene la manzana

5 Por otro lado, en una carta posterior de 1799, Kant rechaza la filosofía de Fichte como criticismo. Véase Moledo, La declaración pública de Kant contra Fichte, 2007. 
delante de la boca, pero no permite que se la pruebe. Se reduce a la pregunta: ¿mundus ex aqua? Él permanece siempre en lo general, nunca da un ejemplo $y$, lo que es peor, no puede dar ninguno, porque aquello que se ajusta a sus conceptos generales no existe (p. 89).

La crítica es similar a la que más adelante le lanzará Hegel (2010): "[h]acer pasar su absoluto por la noche en la que, como suele decirse, todos los gatos son pardos, es la ingenuidad del vacío en el conocimiento" (p. 71). Para Kant, la filosofía de Fichte quiere poner "ante los ojos" la manzana, pero tal evento no cumple la exigencia de la presencia (lo cual sería discutible, porque la manzana está presente).

Por consiguiente, el intento de Fichte de hacer una filosofía acorde al idealismo trascendental se queda en un intento, si somos kantianos rigurosos (en cuyo caso, no se entendería bien qué es eso de la doctrina de la ciencia). Pero, por otro lado, si la pretensión de Fichte era inaugurar un camino nuevo para el filosofar, que tuviera la presencia fundamentada desde el ser, lo consigue. No en balde es el creador del idealismo. Para el idealismo alemán la cuestión del fenómeno como presencia es un problema perpetuo que resuelve Fichte de esta forma. Sin embargo, también Schelling y Hegel tendrán que justificar su filosofía de cara a la presencia.

\section{Referencias}

Derrida, J. (1989). Márgenes de la filosofía. Madrid: Cátedra.

Descartes, R. (1995). Los principios de la filosofia. Madrid: Alianza Universidad. Díaz, J. A. (Ed.) Correspondencia. Kant, Fichte, Schelling, Hegel. Bogotá: Universidad Nacional de Colombia, 2011.

Fichte, J. G. (1975). Doctrina de la ciencia. Buenos Aires: Editorial Aguilar.

Fichte, J. G. (1982). Reseña de Enesidemo. Madrid: Hiperión.

Fichte, J. G. (1997). Introducciones a la doctrina de la ciencia. Madrid: Tecnos. Hegel, G. W. F. (1993). Fundamentos de la filosofía del derecho. Madrid: Libertarias/Prodhufi. 
Hegel, G. W. F. (2010). Fenomenología del espiritu. Madrid: Abada.

Heidegger, M. (2001). Introducción a la metafísica. Barcelona: Gedisa Editorial. Kant, I. (1998). Crítica de la razón pura. Madrid: Alfaguara.

Leibniz, G. (1940). Principios de la naturaleza. Buenos Aires: Editorial Tor.

Pardo, J. L. (2004). La regla del juego. Sobre la dificultad de aprender filosofía. Barcelona: Galaxia Gutemberg.

Rivera de Rosales, J. (2011). El punto de partida de la metafisica trascendental. Madrid: Ediciones Xorqui. 